\title{
Analysis of Animal Model of Acute Pharyngitis Based on Clinical Characteristics of TCM and Western Medicine
}

\author{
Bing-jie $\mathrm{CHANG}^{1}$, Ming $\mathrm{BAI}^{2}$ and Ming-san MIAOa, ${ }^{\mathrm{*}}$ \\ $1,2,{ }^{*}$ Henan University of traditional Chinese medicine, Zhengzhou 450008, China \\ amiaomingsan@163.com \\ ${ }^{*}$ Corresponding author
} Keyword: Characteristics of clinical diagnosis and treatment of TCM and Western
Medicine, Acute pharyngitis, Animal model

\begin{abstract}
Objective: To analyze the animal models and applications of acute pharyngitis based on the clinical diagnosis and treatment of Chinese and western medicine. Method: Through consulting the literature of acute pharyngitis in recent years, the characteristics of acute pharyngitis, modern application and related animal models were analyzed. Result: The symptoms of the existing acute pharyngitis animal models are similar to those of the clinical symptoms. Conclusion: The animal model of acute pharyngitis remains to be improved, and the mechanism of drug action should be further explored to provide a more reliable theoretical basis for clinical treatment. Acute pharyngitis is a common and common disease, and is an acute inflammation of the pharyngeal mucosa, submucosal tissue and lymphoid tissue, as well as a part of the upper respiratory tract infection. Clinical manifestations: throat, sore throat, pharyngeal foreign body sensation, swallowing discomfort and other symptoms[1].In recent years, with the deepening of understanding of etiology and pathogenesis of acute pharyngitis with traditional Chinese medicine and Western medicine, Chinese medicine and Western medicine have made good progress in the treatment of acute pharyngitis[2,3].
\end{abstract}

\section{Chinese Medicine Treatment of Acute Pharyngitis}

At present, the etiology and pathogenesis of TCM pharyngitis at present there is no clear conclusion, but recognized mainly due to lung and kidney, feel invaded by evil poison from the nose and mouth, heat dampness, heat and phlegm fire caused by [4].Hot wind evil poison by the nose and mouth and into, first of all make lung: portal for the lung and stomach, throat first when its harm, invaded and pharyngeal blood and muscle, swelling, pain, burning, or itching, appears the symptom such as cough, because evil is in the table, so the pharyngeal symptom is lighter; Evil heat pass, lung and stomach heat, the steam in the throat, then the throat severe swelling and pain, difficulty swallowing, jaw from the nucleus; Mostly in autumn and winter and winter and winter. Belongs to the Chinese medicine "acute pharyngitis" category, Modern Chinese medicine mainly divides it into: Yin deficiency, fire hyperactivity, yin deficiency, lung dryness, spleen deficiency, weak Qi, qi stagnation and blood stasis, kidney yang loss. Treatment principle should be based on heat. Chinese medicine treatment of pharyngitis diseases has a long history, from the "Shanghanzabinglun" by the "White Tiger Decoction" is the representative prescription treatment of Yangming syndrome of Qi Fen fever, Jiawei Scutellaria, mint, licorice, and effective in the treatment of acute pharyngitis caused by pathogenic wind into the inside of the fire of the lung and stomach heat. The Chinese have throat oral diseases all belong to fire ", traditional Chinese medicine has good curative effect for the treatment of acute 
pharyngitis, such as purging fire detoxification, nourishing lungs, dispelling wind and relieving sore throat treatment of acute pharyngitis [5], The treatment of TCM has many targets and many ways, and it has a good cure rate, low cost and little adverse reaction to acute pharyngitis [6].

\section{Western Medicine Treats Acute Pharyngitis}

Modern medical research shows that acute pharyngitis is by influenza, parainfluenza virus, coxsackie virus, adenovirus, rhinovirus, staphylococcus aureus, group a streptococcus, pneumonic diplococcus, such as viruses and bacteria infections or high temperature, dust, smoke and other physical or chemical factors stimulate the pharyngeal mucosa caused by acute inflammation[7].Currently, western medicine treats acute pharyngitis mainly to fight infection, including the use of antibiotics and anti-virus, as well as the use of antipyretic analgesics, glucocorticoid, tonic, etc., the overall curative effect is certain[8].In clinical use, there are drug resistant bacteria Strains, adverse reactions or medication contraindications affect the efficacy, and sometimes the symptoms are not satisfactory in a short period of time[9].Therefore, the combination of drugs to treat acute pharyngitis has a better effect. Currently, the commonly used western medicine has receptor antagonists, antihistamines, antitussive drugs [10], etc.

\section{The Diagnostic Criteria of Acute Pharyngitis in the Clinic of Chinese and Western Medicine}

\section{Diagnostic Criteria of Traditional Chinese Medicine}

In the treatment of acute pharyngitis, TCM syndrome differentiation is divided into the external wind fever, the external wind chill syndrome and the pulmonary stomach heat. The standard adopts the diagnosis and treatment guide for the common diseases of the ear and throat of Chinese medicine. Respectively, exogenous wind-heat disease: (1) sore throat, throat burning, fever, (2)headache, cough, sputum; (3)pharyngeal mucosa bright red and swollen; (4tongue side red, thin white fur, pulse floating number; (1) sore throat, mouth thirsty, aversion to cold, (2)headache, cough, sputum thin; (3pharyngeal mucosa color red and swollen; (4)light red, thin white fur, pulse floating; More swollen, swallowing difficulties, (2) cough sputum, constipation and urine; (3)pharyngeal mucosa swelling, pharyngeal wall lymphoid follicular swelling, or submandibular lymph nodes; (4)tongue red, moss yellow, pulse number.

\section{Clinical Diagnostic Criteria of Western Medicine}

Western medicine standard is "practical integrated traditional Chinese and Western medicine diagnosis and treatment of acute pharyngitis" diagnostic criteria: (1) more acute onset; (2) sore throat, dry burning, swallowing bad, and even swallowing difficulties; (3) pharyngeal swelling, pharyngeal wall lymphatic filtration Swollen and yellow and white point-like exudates, pharyngeal staggering and uvula edema, and even pharyngeal rostra swelling, both sides of the lower angle of lymph nodes and tenderness; (4) have chills, fever, headache, general malaise Etiology; (5) blood test the total number of white blood cells increased. 


\section{The Characteristics of Acute Pharyngitis Animal Model, the Indexes of Detection and the Agreement with the Clinical Diagnostic Indexes of TCM and Western Medicine Were Analyzed}

Animal models of acute pharyngitis usually choose rats, rabbits and so on. Among them, rats have the advantages of pure, large quantity, low price and so on. They are the most commonly used [11].Pharyngitis is more common in adults, usually adult animals. In the experimental animal model of acute pharyngitis, an animal model can be made according to the cause of the disease. The main methods of replicating animal experimental models can be divided into bacterial infection, physical and chemical stimulation, and other methods [12].

\section{Bacterial Infection}

The acute pharyngitis model was established by injection of bacterial infection. The pathogenic factors were the same as those of clinical acute pharyngitis. The characteristics of the model were similar to that of the clinical and were commonly used in the experiment. Experimental animal pharyngeal redness, infected mucosa with punctate purulent secretions, or epithelial keratinization serious spikes, glandular hypertrophy and hyperplasia, mucosa, submucosa, lamina propria muscularis stroma showed obvious inflammatory cell infiltration. This method requires two infections after $24 \mathrm{~h}$. The model of acute pharyngitis established by bacterial infection is shown in Table 1.

Table 1 Establishment of acute pharyngitis model by bacterial infection

\begin{tabular}{|c|c|c|c|c|}
\hline $\begin{array}{l}\text { Building } \\
\text { methods }\end{array}$ & $\begin{array}{l}\text { Building } \\
\text { animal }\end{array}$ & $\begin{array}{l}\text { Mecharism and } \\
\text { characteristics }\end{array}$ & $\begin{array}{c}\text { The clinical } \\
\text { symptoms } \\
\text { were } \\
\text { consistent with } \\
\text { the diagnostic } \\
\text { criteria }\end{array}$ & $\begin{array}{l}\text { Matters needing } \\
\text { attention }\end{array}$ \\
\hline $\begin{array}{l}\text { Type b hemolytic } \\
\text { streptococcus } \\
\text { was injected into } \\
\text { the pharyngeal } \\
\text { mucosa and } \\
\text { established an } \\
\text { acute pharyngitis } \\
\text { model }\end{array}$ & Rat & \multirow{2}{*}{$\begin{array}{l}\text { Bacterial infection } \\
\text { of the } \\
\text { pharynx,causing } \\
\text { acute infammation. } \\
\text { Advantages:similar } \\
\text { to human disease } \\
\text { mechanism,pathog } \\
\text { enic factors and } \\
\text { clinical acute } \\
\text { pharyngitis.In the } \\
\text { same } \\
\text { way,representation } \\
\text { is similartoclinical } \\
\text { practice }\end{array}$} & $\begin{array}{l}\text { And clinical } \\
\text { indicators } \\
\text { consistent: in } \\
\text { line with } \\
\text { Western } \\
\text { indicators } \\
\text { (1) (2) (3), } \\
\text { anastomosis } \geq \\
80 \% \text {. }\end{array}$ & \multirow{2}{*}{$\begin{array}{l}\text { Model animals } \\
\text { undergo a second } \\
\text { pharyngeal } \\
\text { mucosal bacterial } \\
\text { infection after } 24 \\
\text { hours [13-15]. As } \\
\text { the animals } \\
\text { infected with } \\
\text { pathogens have a } \\
\text { certain infectious, } \\
\text { in the course of the } \\
\text { experiment should } \\
\text { be avoided by } \\
\text { experimental } \\
\text { animals. }\end{array}$} \\
\hline $\begin{array}{l}\text { Staphylococcus } \\
\text { aureus was } \\
\text { injected into the } \\
\text { pharyngeal } \\
\text { mucosa to } \\
\text { establish an acute } \\
\text { pharyngitis } \\
\text { model }\end{array}$ & Rats, rabbits & & $\begin{array}{c}\text { In line with } \\
\text { Western } \\
\text { indicators (1) (2) } \\
\text { (3) 5), } \\
\text { anastomosis } \geq \\
80 \% .\end{array}$ & \\
\hline
\end{tabular}

\section{Physical or Chemical Factors to Stimulate}

Acute pharyngitis is a common and frequently occurring disease in clinic. Irritant 
chemical gas is one of the pathogenic factors. Ammonia water was stimulated by direct spraying and fixed point quantitative smearing method. Rats or rabbits were selected as experimental subjects, and the animal models of acute pharyngitis were developed by spraying ammonia water in the throat. Ammonia is an alkaline irritant, high concentration local spray or fixed-point quantitative coating can stimulate pharyngeal mucosa, so that congestion, swelling, forming acute inflammation. Physico chemical factors for the establishment of acute pharyngitis models are shown in table 2 .

Table 2 Establishment of acute pharyngitis model by physical and chemical factors

\begin{tabular}{|c|c|c|c|c|}
\hline $\begin{array}{l}\text { Building } \\
\text { methods }\end{array}$ & $\begin{array}{l}\text { Building } \\
\text { animal }\end{array}$ & $\begin{array}{l}\text { Mechanism and } \\
\text { characteristics }\end{array}$ & $\begin{array}{l}\text { The clinical } \\
\text { symptoms were } \\
\text { consistent with the } \\
\text { diagnostic criteria }\end{array}$ & $\begin{array}{l}\text { Matters } \\
\text { needing } \\
\text { attention }\end{array}$ \\
\hline $\begin{array}{c}\text { An animal } \\
\text { model of acute } \\
\text { pharyngitis was } \\
\text { induced by } \\
\text { ammonia water } \\
\text { stimulation in } \\
\text { the pharyngeal } \\
\text { mucosa of rats. } \\
{[16]}\end{array}$ & $\begin{array}{l}\text { Rats, } \\
\text { rabbits }\end{array}$ & \multirow{2}{*}{$\begin{array}{c}\text { Physical and } \\
\text { chemical } \\
\text { stimulation, so that } \\
\text { the pharyngeal } \\
\text { mucosa due to acute } \\
\text { stimulation and } \\
\text { congestion swelling, } \\
\text { the formation of } \\
\text { acute inflammation. } \\
\text { Advantages: simple } \\
\text { and safe method of } \\
\text { operation, } \\
\text { characterization and } \\
\text { pathological } \\
\text { conditions and } \\
\text { clinical similarity }\end{array}$} & $\begin{array}{c}\text { Consistent with } \\
\text { Western indicators } \\
\text { (1) (2) (3) (5), } \\
\text { anastomosis } \geq 90 \% \text {; } \\
\text { in line with } \\
\text { traditional Chinese } \\
\text { medicine exogenous } \\
\text { wind and heat } \\
\text { syndrome (1) (3), } \\
\text { anastomosis } \geq 70 \% \text {. }\end{array}$ & \multirow{2}{*}{$\begin{array}{l}\text { The use of } \\
\text { ammonia } \\
\text { water } \\
\text { stimulation, } \\
\text { the amount } \\
\text { and location } \\
\text { of small } \\
\text { animals is not } \\
\text { good control, } \\
\text { and may be } \\
\text { due to } \\
\text { improper } \\
\text { operation, } \\
\text { causing local } \\
\text { infection of } \\
\text { animals, lung } \\
\text { and stomach } \\
\text { burns. }\end{array}$} \\
\hline $\begin{array}{l}\text { Ammonia water } \\
\text { fixed point } \\
\text { quantitative } \\
\text { smear rabbit } \\
\text { throat to } \\
\text { establish acute } \\
\text { inflammatory } \\
\text { animal model } \\
{[17]}\end{array}$ & Rabbit & & $\begin{array}{c}\text { Consistent with } \\
\text { Western indicators } \\
\text { (1) (2) (3) 5), } \\
\text { anastomosis } \geq 90 \% \text {; } \\
\text { in line with } \\
\text { traditional Chinese } \\
\text { medicine exogenous } \\
\text { wind and heat } \\
\text { syndrome (1) (3), } \\
\text { anastomosis } \geq 70 \% \text {. }\end{array}$ & \\
\hline
\end{tabular}

Important Indicators of Acute Pharyngitis [18, 21]

Pathological Indicators. The connective tissue, vessels and submucosal glands of the pharynx were observed. To observe the rat pharyngeal mucosa under light microscope, whether the emergence of a large number of epithelial hyperplasia, showing top dendritic, lamina propria edema, infiltration of inflammatory cells such as monocytes, lymphocytes and eosinophils, vascular proliferation, wall thickening, expansion and hyperemia, connective tissue thickening, muscle fiber disorder, gland enlargement of mucosa integrity, increased secretion of inflammatory cells in the cavity.

Apparent Index. The color of pharynx, the redness and swelling area of the pharynx, the shedding of the mouth and the skin, the secretion of saliva, the luster of the coat, the situation of cough and asthma, and the activity of the pharynx were observed. Pharynx appears large area redness and swelling, mouth fur off, saliva secretion increase, appear cough and asthma phenomenon.

Biochemical Index. Serum levels of TNF- alpha, IL-1 beta, IL-6 and so on were detected. Enzyme linked immunosorbent assay (Elisa Kit) was used to detect inflammatory factors, interleukin -1 and interleukin -6 in serum. 


\section{Conclusions}

The mechanism for clinical treatment of acute pharyngitis mainly through the throat, thirst, cough, phlegm, relieve pain and inflammation in the tissue damage and fibrosis, reduced endothelial cell contraction and endothelial cell injury, expression, regulation of cytokine inhibition of pharyngeal pathological morphology, inhibiting the over expression of IL-1 beta, IL serum -6, TNF- alpha, improve immune function etc..The theory of Chinese medicine of acute pharyngitis is mainly due to lung and kidney, Waixie, toxin from the nose and mouth, heat dampness, phlegm heat caused by fire. The main symptoms are redness and swelling of the throat, pain and other symptoms $[22,23]$. This is in accordance with modern medical understanding of acute pharyngitis.

According to the analysis of acute pharyngitis and clinical symptoms and signs consistent analysis of the situation, the main indicators of efficacy including sore throat, throat burning, pharyngeal mucosal hyperemia; secondary endpoints included fever, cough, throat itching throat lymph follicles. Clinical efficacy evaluation with reference to "Chinese Journal of Otorhinolaryngology common diagnosis and treatment guidelines", "Chinese medicine diagnosis criteria" [24], "Chinese medicine new drug clinical research guidelines" [25], "clinical diagnosis and efficacy judgment of the four weighted score method Introduction, "and clinical symptoms and signs in accordance with its severity is divided into four levels, mainly with the clinical symptoms and signs of symptoms and signs of $0,2,4,6$, secondary and clinical symptoms and signs of symptoms of $0,1,2,3$ [26].

In the acute pharyngitis model, rats or rabbits were used as experimental subjects. According to the medical science research, the adult animals were usually used in the acute pharyngitis model. The reason is that acute pharyngitis usually occurs in adults. Taking adult animals as the object of study, there are many kinds of existing acute pharyngitis models, and the method is reliable and reproducible. In the model by injection of oral streptococcus is ideal, this is because of acute pharyngitis mostly bacterial and viral infections [27], bacterial infection with Streptococcus, Staphylococcus aureus and Streptococcus pneumonia, of which group A streptococcal infection was the most serious, hemolytic streptococcus for clinical types the patients with acute and chronic pharyngitis in the highest detection rate of bacteria, accounted for $54.4 \%$.Using this modeling method, the pathologic characterization of model animals is similar to that of clinical acute pharyngitis, and the symptoms and signs are similar to the diagnostic criteria for the clinical study of acute Chinese medicine for the treatment of acute pharyngitis. Staphylococcus aureus injection model animal oral, causing infection, and human pathogenesis similar, but because the infected pathogens of animals with a certain degree of infectivity, the experimental operator will have the risk of infection, the experimental safety requirements are higher. The establishment of animal model of acute pharyngitis stimulation in the physical or chemical method, and the model of local clinical acute pharyngitis have similar changes, animal pathological injury and serum cytokine index model has certain similarities with clinical indicators, and the operation is simple and safe, although the relatively large animal damage, prone to oral mucosa burns or lung damage phenomenon. It may be because the experimental animals are struggling with accidents, causing injuries to the mouth and stomach, or because of a fault that causes an infection in the injured area, but it is still a widely used model building method. The experimental model of acute pharyngitis animal pathogenesis can very well help people further study of acute pharyngitis, curative effect and mechanism of evaluation for treatment of acute pharyngitis, and provide experimental basis for the clinical treatment of acute 
pharyngitis [28].Therefore, it is the next step to explore the pathogenesis of acute pharyngitis and establish a scientific and stable animal model of acute pharyngitis.

\section{Acknowledgement}

This research was financially supported by the National International Cooperation Base (external letter 2016-65), Zhongyuan scholar (162101510003), Henan Province Production and Research Project (142201310013 172107000012)

\section{Reference}

[1] Ma Jianli. Bianyan oral liquid on acute pharyngitis animal model of curative effect and LI-1 beta, IL-6, TNF- expression [A]. China Pharmaceutical Association, the Jiangsu Provincial People's government.2012 Chinese Pharmaceutical Conference and the twelfth China essays on the [C]. China pharmacy pharmacist week, the people's Government of Jiangsu Province: 2012:5.

[2] Yan Jihong. [J]. Chinese experience of continuing medical education, 2015 clinical treatment of acute pharyngitis with combination of TCM and Western medicine, (33): 221-222.

[3] Zhou Ling, Zhang Jingfei. TCM treatment of acute pharyngitis clinical research progress of traditional Chinese medicine [J]. journal, 2012, (04): 135-137.

[4] Wang Xiaoxiao, Yancang Yan, Wang Xudan, Ping Jiayi, Duan Xiaoying. The anti-inflammatory effects of compound niubangzi buccal tablets and its therapeutic effect on acute pharyngitis in a rat model of $[\mathrm{J}]$. Chinese experimental pharmacology journal, 2010, (11): 147-149.

[5] Ma Qianrong, Wang Wenzhi, Wei Yuxing, Pan Jishan, Zhang Ming, Huang Qin Qing Decoction for the treatment of acute pharyngitis of wind heat type [J]. Asia Pacific traditional medicine, 2017, (10): 134-135.

[6] Li Ruilin, Wang Fang, Zhao Fang, Yang Weizhuo. TCM Decoction for treating acute pharyngitis clinical effect of [J]. Chinese contemporary medicine, 2016, (15): 97-99.

[7] Xue Shuyuan, Zhu Dan, Yao Xingfeng, Jia Qi, Wang Dongrui, Li Chunyan. Survey of animal experiments on acute and chronic pharyngitis [J]. Chinese Academy of medicine, 2016, (10): 121-122.

[8] Guiqing. Metronidazole and penicillin treatment of acute tonsillitis clinical efficacy of [J]. Medical forum, 2012 (08): 996-997.

[9] Beauty show, Wang Zhiyi. Experimental study on [J]. TCM Yin in rats with acute pharyngitis. Journal of Wuhan University (Medical Science Edition), 2014, (03): 366-369.

[10], Wang Chunyan, Wen Xiaohui, Liu Jinfeng. Leukotriene receptor antagonist combined with antihistamines in the treatment of allergic pharyngitis [J]. Chinese Journal of clinicians, 2014, (23): 4298-4301.

[11] Liu Xueyan, Mao Zhigang, Wang Ping, Gao Yan, Zhang Huayun. Study on the anti-inflammatory mechanism of Yanyan drop pills in rats with acute pharyngitis [J]. Pharmaceutical research, 2016, (06): 319-321. 
[12] Tax Lei, yellow star, Huang Heng. Journal of Otorhinolaryngology, combined with clinical observation of HOUYANQING buccal tablet combined with Iodine Glycerol $[\mathrm{J}]$. Chinese treatment of acute pharyngitis of traditional Chinese medicine and Western medicine 2016 (02): 108-111.

[13] Chenfeng, Cao Liang, Kou Junping, Li Na, Heng Frances, Ding Gang, Wang Zhenzhong, Xiao Wei. Acute pharyngitis Danmu leaves in rats to study the effects of [J]. China clinical laboratory science journal, 2015, (23): 97-100.

[14] Liang Lu. Effect and mechanism research of Shanxi University [D]. compound of Liyan Oral Liquid on experimental acute and chronic pharyngitis, 2012.

[15] Liang $\mathrm{Lu}, \mathrm{Pu}$ Jinhua, Ma Jianli. Study on pathogenesis and animal models of acute and chronic pharyngitis [J]. Clinical medical practice, 2012, (07): 540-543.

[16] Wang Jun. Experimental study on the effect of Herba Houttuyniae Injection on acute pharyngitis [J]. Chinese Pharmaceutical Science, 2016, (03): 35-37.

[17] Tian Huajie, Huang Xiaoxing, Wang Yifei, Xu Xiaotao, Sun Haiyan, Liu Li. Model of acute pharyngitis rabbit model establishment of [J]. world clinical drugs, 2014 (04): 241-244.

[18] Zhang Li, Wang Baozhong, Bai Yinfu. Effect of Yinhuang buccal tablets on chronic pharyngitis rabbit model of $\mathrm{C}$ - reactive protein and interleukin -6 expression [J] Chinese medicine, 2008, 12 (3): 742-744.

[19], Zhang Li, Yang Jun. Expression and significance of MM, P-2 and CRP in pharyngitis animal model $[\mathrm{J}]$. Chinese Journal of experimental and infectious diseases, 2009, 3 (3): 259-264.

[20], Peng Shunlin, Zhang Yue, Chen Juan. The influence of oral pharynx liquid on the expression of IL-1 beta and EGF in experimental chronic pharyngitis [J]. Chinese Journal of integrated traditional and Western medicine, otorhinolaryngology, 2008, 16 (3): $168-170$

[21] ho Liang Qiang Liu Yuan Xian, Yang Long, Wu Xinfeng, and even profit, $\mathrm{Hu}$ Zhen, Liu Yuanyuan, Xiong Yalan. To establish the animal model of chronic pharyngitis and the related indices with otorhinolaryngol, [J]. China analysis of traditional Chinese medicine and Western medicine 2017 (01): 17-20.

[22] Wang Wenping, Zhao Keming, Gao Jingjing, Lv Yuping, Yu Ming. The etiology and pathogenesis of acute pharyngitis theory $[\mathrm{J}]$. Liaoning Journal of traditional Chinese medicine, 2009, (07): 1086-1088.

[23], Sun Mingjuan, Huang Chunjiang, Li Yunying, Qiao Fengying, Chen Xiaoning, Shen Hui, Gan Jing, Bing Mei pian. A multicenter randomized controlled study on acute pharyngitis of wind heat and lung type [J]. Chinese Journal of traditional Chinese medicine, 2017, (01): 38-41.

[24] Zhao Junjie. Clinical observation on treatment of acute pharyngitis and acute suppurative tonsillitis in children with open throat sword spray [J]. new Chinese medicine, 2014, (10): 147-149.

[25] Yu Jiaojiao, Xuan Zhenyu, Zhang Huiyong, Shi Kehua, Ruan Yan, Guo Yu. HOUYANQING buccal tablets in the treatment of acute pharyngitis clinical study of [J]. China Journal of TCM, 2015, (02): 351-355. 
[26] Hu Rong, Wang Lihua, Zhang Junjun, Guo Yu, Xiao Feng Jie Du capsule in the treatment of acute pharyngitis with wind heat syndrome [J]. drug evaluation study, 2014, (05): 460-462.

[27] Wang Bingnan, Zhang Peisen, Tan Yongli, Gao Xiaohua. Dexamethasone combined with Houttuynia ultrasonic nebulization in treatment of acute pharyngitis clinical observation [J]. Hebei medical journal, 2014, (02): 266-268.

[28] Liang Lu, Pu Jinhua, Gao Tianhong, taekang. Study to evaluate the efficacy and mechanism of $[\mathrm{J}]$. Drugs on experimental acute pharyngitis animal model of the Potassium Sodium Dehyd roand rographolide Succinate for Injection 2012, (03): 165-168. 